\section{TREATMENT OF CONSTIPATION.'}

\section{BY IENRY M. FIELD, M.D.,}

OF noston, MAss.

The physiological resources-or, better still, the resources answering to physiological indicationwhich the physician has at his command for the relief and cure of chronic constipation, may, for purposes of analysis and classification, be referred to three subdivisions.

First.-Chronic constipation is quite often habitual constipation, and is to be most effectually combatted by habitual and systematic attention to the demands of nature in a periodic evacuation of the bowels. Success along this line involves exceptional intelligence, and resolution; and confidence, on the part of the patient. The poet Cowper has well ex. pressed the difference of task in the formation of a habit and reformation in the stanza-

\section{" IInbits are soon assumed; but when we strive} To strip them, 't is being flayed alive!"

It is far easier to swallow an occasional or a frequent pill than it is to adopt the system required for the removal of a bad habit in respect of constipation; and the patient, whose interest and confidence can be enlisted at first, too often loses faith in herself or the system, and, indeed, in the doctor. The young woman who has been so badly trained in her maternal home-and cases of this kind, througlout our best families even, are innumerable-that she has no other notion of the natural functions of the bowels than that response is to be made only to an emphatic call, an experience which may happen once in three or four days, or once a week, or less often, and who receives as a new revelation the statement of her physician that there should be alvine evacuation daily, at a regular hour, and the most rigid system observed in this particular, has no light occupation before her in an effort to inaugurate reform after so many years of inattention and irregularity. But such task has been repeatedly accomplished upon the condition of a fairly good constitution, of certain measures chiefly dietetic and hygienic of help, and of determined perseverence; and constipation thus cured is effectually and permanently cured.

The patient is to be instructed to select that hour of the day hest in consonance with previous habits and least liable to interruption, ${ }^{2}$ and invariably, as the hour comes round, to make an effort at stool, even though there be no solicitation and notwithstanding there should be frequent insuccess. So inevitably is man the creature of habit in every realm of his being, that the patient who invariably and resolutely attempts to perform any physiological function at a stated hour every day, will very surely, after a time, carry out his purpose; and the more especially in the present instance if he have every assist-

\footnotetext{
1 Read before the Gynacological Society of Boston, May 20, 1886

2 Notr.-The hour best in consonance with previous habits, etc. Not necessarily at carly morning lour, although such is doubtless the hour observed by the majority of those who have established a habit of regularity. A short-sighted effect to force a particular hour upon a paof the day, bas becu sufficient to bring discouratement and failure in some instances known to the writer.
}

ance from a carefully adapted regimen. Of course, any physiological law whatever must have intelligent interpretation; and allowance must be made for occasional departure from the usual standard. There are patients who have excellent health upon the basis of a regular movement of the bowels once in fortyeight hours; and my preceptor, Dr. Peaslee, used to report the case, observed in one of his families, where both husband and wife, then arrived at old age, had each been accustomed for many years, if not through life, to evacuate the bowels only once in nine days! It is the principle of regularity that is to be insisted upous; and a single occasion of failure or neglect, in an individual whose bad habit of constipation had been largely corrected, may cause a serious relapse into the old and depraved method, or lack of method, of life.

Details can hardly be expected from a paper which is confessedly but fragmentary and suggestive; it is to be understood that the regimen which is to assist the patient in the formation of a new habit, shall receive the largest construction and be the object of a special application in every case. Whatever best offers assistance, whether from the department of hygiene or dietetics, is to be laid under contribution. Such appliances as the electric battery, the electic belt, localized massage, kneading of the bowels, etc., have a high and, indeed, an essential value in some instances.

But all cases of habitual constipation, as already intimated, are not susceptible of cure by the scheme of the formation of a new and correct habit; and this for various reasons; probably those cured and capable of cure, at least as found in adult life, are quite exceptional. The patient cannot and will not per. severe in the necessary effort; the bowels, long inused to overdistension and torpor, will not respond to the most positive of alimentive stimuli; help must be had from medicine, and this medicine drawn from the class of cathartics. What is a cathartic? I have frequently found occasion to say that the definition of anything pertaining to special therapentics must squarely meet three conditions, if it is to be depended upon to convey the requisite information: It must be intelligible and concise and comprehensive. Judged by such standard, I know not where in any of all the books devoted to therapeutics, we shall find a satisfactory definition of cathartic. Fothergill approaches the requirement of the case, and yet his terms are not quite free from fault. The following, therefore, is proposed for approval or criticism: $A$ cathartic is an agent or agency which increases both peristalsis and intestinal secretion-either element of action, ordinarily, being prominent according to the material used-with result of provoking preternaturally free and frequent evacuation of the bowels.

'There is no need to remind you of recent physiological experiments which have established the fact that every agent which acts cathartically, even though the influence be of that mild degree recognized as laxative, does so by procuring both hypersecutive and increase of contractility. One agent acts chiefly on the glandular structure and the stools will be watery; another acts mostly through energized per- 
istalsis, and the dejections are feculent, as with Epsom salts in the former case, and senna and aloes in the latter. There is one purgative, and perhaps only one, catharsis from which appears to present equivalent of action, viz., castor oil ; a consideration which, together with the conjoined fact of nearly equable distribution of influence throughout the entire intestinal tract, renders this offensive medicament too often essential to the physician. With an occasional laxative in an occasional subject, energized contractility may be so little pronounced that the serous effusion evoked may remain in the bowel-unless reabsorbed-until some peristaltic is administered to procure its evacuation. But none the less is it true physiologically that the two elements specified always inhere to cathartic medication, and, in variable degrees, are always present in the operation of a cathartic remedy; and the recognition of this princi. ple is essential to the successful employment of this class of materials.

Again, cathartics vary materially in their action regionally considered; $e$. gr., senna acts chiefly on the smail intestines and aloes on the lower bowel. Some, like jalap, and especially elaterium, act so high up as, frequently, to involve the stomach in the irritation produced and occasion nausea, and even vomiting. It should be borne in mind that the selection of a cathartic, with respect of its operation cathartically upon the small or the large bowels, must have a very important bearing upon the alimentation and nutrition of the patient; for a cathartic which acts energetically upon the upper bowel and so sweeps it of nutritive material ipon which the energies of digestion have been already expended, and which is in situ and in process of and for assimilation, depresses the patient far more seriously than does the emetic, which simply evacuates the stomach of undigested or indigestible food; while, per contra, the other purgative, which gives its force only to the larger bowel, can bring away little more than residual and excrementitious matter.

Still another fact must receive the attention of the physician who expects to employ a cathartic safely and effectively. Some purgative materials exert an influence, near or remote as concerns other organs, of which it is not practicable to take account in any definition of catharsis. In addition to modified action in the function of muscilar and glandular structures, rhubarb and magnesia, prominently, effect important changes in the morbid condition of the mucosa of the intestine; and the fact that mercury is indicated in certain functional disturbances of the liver, and that aloes is contraindicated in morbid lesion of the rectum and uterus-familiar to every physician-are sufficient illustrations of the principle which we would emphasize.

Each subject of chronic constipation, requiring help from cathartic medication, must be regarded as a special problem, and, as it were, to be carefully solved before effectual aid can be afforded. An attempt to apply the methods of a routine practice will quite surely bring disaster or disappointment. The physiological characteristics of the prominent cathartics being already understood, it is now to be

determined what are the individual peculiarities of the patient who presents himself for treatment. Is the constipation to be traced to inertia of muscle? and is this located chiefly in the large or small bowel? Or, again, is the prominent defect referable to a generally defective secretion from the glandular apparatus, or perhajs the entire camal? If it is that great gland, the liver, withont the normal working of which no important task of either stomach or bowels can be healthily performed, if it is the liver that is at fault, it should go without the saying that no therapentic measures directed against constipation can be really remedial which do not ensure the return of the bile, in proper quantity and quality, to the intestine. The researches of Rutherford, in particular, among recent physiologists, have provided us with new materials for service in this direction; but all the negative conclusions of all the physiologists from the time of Bennett have not availed to release the clinical physician from primary, if not inevitable, dependence upon mercury, in all conditions which unmistakably indicate recourse to a cholagogue.

If it is a weak and anemic woman, whose symptoms point to torpor located in the small intestine, it will be a difficult task so to adjust medication as to restore activity, procure systematic evacuation and yet not seriously to deplete. On the other hand, the individual who habitually over-eats and who is consecquently plethoric and perhaps bilious and constipated, will, in addition to a timely mercurial, find an admirable resource in the frequent use of the mildly saline cathartic waters, such as hunyadi, etc., so popular now-a-days; popular, doubtless, because their special rôle has come to be so generally recognized by the large class requiring such intervention. At the same time, it may be questioned whether the practice of the Roman epicure, in the employment of the emetic, as Seneca gives it-" They vomited that they might eat, and they eat that they might vomit," was not more physiological, as it was surely more direct in its purpose and results.

Probably no one material belonging strictly to the class of purgatives is so valuably to the physician, in the treatment of chronic constipation, as is nux vomica, classified elscwhere; and because whatever other element may be present, a deficient tonicity is quite sure to complicate the worst cases of this affection. Nux vomica is the great neurotic energizer of digestion; it is to the nervous element in digestion what pepsin is as a catalytic, and it is the great peristaltic. Many cases can be successfully treated without help of other medicine than nux vom., through dependence upon the measures specified in the first section of this paper. Still further, the consideration that nux vom. has much of the stomachic properties of the simple bitter, combined with the component power of quinia, renders it the more valuable in such application. Again, nux vom. is valuable because the energy for specifically peristaltic cathartic is much enhanced by its combination; so that, $e$. $g$., the fraction of a grain of aloes may prove as effective in union with nux vom. as would a large integral dose of aloes alone.

Once more, the elimination, by the pharmaceutical 
chemist, of the active principles of aloes is a fact of much importance in the treatment of habitual constipation; so far, at least, as aloetics are applicable. Thereby much greater concentration is secured, and not this alone, but the essential oil, which is largely responsible for the nauseous smell and taste of aloes, is avoided, and it is no longer obligatory upon the physician, in his offer of a purgative, to present a bulky and offensive medicament. A parvule constituted of aloin and nux vomica, aa gr. $1 / 3$; or of aloin, gr. $1 / 3$, strychnia sulphate, gr. $\frac{1}{30}$, with addition of gr. $i$ ex $\mathbf{j}$ hyoscyamus, to be taken after meals once or thrice daily $p r . r . n$, provides a resource more generally appropriate than perhaps any other that can be devised.

But aloetics have a wide and varied range of contraindication; it is pretty surely true, notwithstanding the assertion of some authorities to the contrary, that habitual dependence upon the smallest dose of aloes will gravely increase any active disease of either rectum or uterus. For the subject of chronic constipation, with complicating hemorrhoids or metrorrhagia, or uterine inflammation, it is a difficult thing to find a substitute for the aloetic pill. But the introduction of cascara sagrada has rendered the task easier, and a pill made of nux vom. with gr. iij ext. cascara sagrada will sometimes meet the latter indication admirably.

It is an accepted statement with the therapeutic physiologist that physostigma increases secretion along the course of the alimentary canal; and physiologically considered, there should be provicled a mild laxative in a combination of nux vomica and physostigma, as setting in active operation the two essential factors of a cathartic influence. At all events, physostigma introduced into the aperient pill should meet a corresponding indication, as respects the glandular structure of the bowel, with nux vom. in its action upon the muscular coat. From my own limited clinical experience with the former remedy, I have failed thus far to be satisfied of this fact, but in the subject of chronic constipation with prevalently dry dejections, it would receive the sanction of the authorities to combine a fraction of a grain of physostigma with the ingredient of the aperient pill.

Finally, idiosyncrasy is the occasion of much concern in the systematic and habitual use of cathartic remedies; - it is doubtful if individual peculiarities assert themselves more arbitrarily in reference to neurotic remedies than in such employment of cathartics. If the patient is a female, and especially if she has been long pampered by habits of self-indulgence and luxurious living, the case must be very carefully studied upon its own merits and demerits, or the physician will quite surely go wrong. 'The selection of remedies having been made and their apportionment decided upon, it is well that the patient should understand that no two cases of constipation ever required exactly the same treatment, and that quite likely there must be repeated trial and modification of the measure proposed and report to the physician before an exact adaptation can be made in the present instance. I arge experience does not always bring confidence; a large experience in the treatment of chronic constipation rather brings distrust, i. e., to the physician; and it is right that the patient should, at the start, be imbued with a measure of this distrust and acquainted with the reasons for it, alike in justice to the doctor, the system and himself. Many patients will not meet the inevitable conditions-they soon get discouraged or are satisfied with partial results; but when the physician can secure such cooperation, it is assuredly alone his fault if the treatment is not at last reduced to successful terms.

There are, however, some general facts in the employment of special cathartic materials, of which the following must serve as illustrations; and the more of such the practitioner may have at command the less often will he commit a deplorable error:

I. The salines do not commonly agree with the aged-they find them too chilling; and a dose of Epsom salts, which may opcrate very kindly upon the young and middle-aged and vigorous, may bring serious disaster to the old man or old woman. A sudden depression of vital energy and the function of calorification thus procured, together with other favoring circumstances, have more than once precipitated the subject into a fatal pneumonia.

2. All cathartics are apt to be attended with colicy complications when given to a womar at the epoch of menopause; and especial combination at such time, as with comminatives, should be directed against this painful action. Some of the text-books or lectures used to report the case of a young doctor who was dismissed with contumely from a prominent family, upon which he had chiefly depended for the advance of his fortune, because, of offense given to the alimentary canal of a matron, through neglect of this precaution.

3. The common domestic cathartic, senna, should never be prescribed to the subject of cumulative constipation or of impacted faces; if there be anything answerable to a frecal plug formed in the course of the small intestine or near the valve, on either side, such a peristaltic cathartic as senna will infallibly occasion serious and even alarming colic before evacuation can be accomplished; and the same restriction applies to a similar use of an integral dose as calomel. ${ }^{1}$

4. And to make affirmative provision for the subject of the last restriction, in a case of impacted constipation, when it is to be presumed the bowels are more or less distended with hard, dry, knotty, scyabalous masses (and the more imperatively if there be suspicion of complicating typhlitis or perityphlitis), nothing works so safely and so well as Epsom salts, possibly energized with minute doses of tart. emetic, gr. $\frac{1}{\sqrt{6}}$ to $\frac{1}{2}$. Or if it be desirable to apply a cautious peristaltic stimulant in this condition, or in whatever condition declares absence of organic con. tractility in the bowels, nothing else can be so direct in operation as gr. $\frac{1}{70}$ sulph. strychn., inserted once or twice in the twenty-four hours in the areolar tissue of the abdominal wall.

1 Note-An infusion of senna may, however, be administered as en. ema when the torpor and distension are mainly located in the large bowel, both safely and effectually. The excitant influence of senna over organic muscular fibre of bladder, and uterus also, was perhaps more familiar to 
5. There is applarent perplexity in providing cathartic medication, whether on occasions of temporary or habitual constipation, for the subject of a prevalent uterine hamorrhage-i. e., menorrhagia or metrorrhagia. Of course, aloetics are absolutely contraindicated, and senna and cascara no less absolutely; unless, indeed, in the exceptional instance where such a remedy as ergot is reçuired. But there is a cathartic, or laxative cathartic, which deservedly has the reputation of exerting somewhat of inhibitory influence upon hamorrhage from the womb, viz.. cream of tartar. It would be hard to name another which, in its action upon the bowels, arouses so little of peristalsis; and it is not possible that cream of tartar, in any degree of action, should stimulate uterine muscles to contraction; while its tendency to reduce temperature, and especially to lower blood pressure, should work favorably for the arrest of hæmorrhage from whatever cause.

The last section of our subject contemplates, if not the cure of habitual constipation, at least an escape from its discomforts and injurious influence upon health, by the systematic use of water-enemata; but its discussion must be postponed for want of time. It may be said, however, in closing, that loose aud erroneous notions prevail to an extent among the profession even, respecting the value and the consequences of this measure of relief. 'The rectal syringe, like the uterine pessary, may do incalculable mischief; suitably, appropriately, strictly and intelligently controlled, it may be of incalculable benefit. In either instance, there must be selection of cases and considerate adaptation. In no other department of medical practice is the tyro in medicine more out of place, as well as the physician who gives but a passing thought to the condition for which he is called to prescribe.

\section{MEDICAL PROGRESS.}

Placenta Pravia.-At the first meeting of the German Gynecological Association DR. BAYER, of Strassburg read a paper on this subject. Duncan's theory as regards hemorrhages in placenta previa was formulated to apply only to the hamorrhages occurring with the pains and suffices for these alone. Since that time we have learned of the formation of a lower uterine segment. 'This is not simply the lowest portion of the uterus, but a zone, character. ized by quite definite qualities, which does not take part in the contractions, and depends after labor in a condition of extreme relaxation. The hamorrhages have been brought into connection with the development of this lower segment, and since the latter forms during pregnancy, this apparently explains also the hemorrhages occurring during gestation. He then compared the various views respecting the origin of the lower uterine segment with the conditions existing in placenta pravia. According to the views of those who look upon the lower segment as a portion of the body of the uterus, the placenta pravia must be partly or wholly inserted into the segment. But this is a physiological impossibility, at least in cases terminating favorably, for owing to the absence of contraction or retraction of the placental site in the lower segment, every parturient with placenta pravia would be hopelessly doomed to death from hamorrhage. The other view, according to which the lower segment forms from the supravaginal portion, explains the phenomena in a less forced manner. But then the questions arise, why does hamorrhage not invariably occur during pregnancy and why can the placenta be felt occasionally immediately over the closed cervical canal, or one with opened parallel walls? These objections cannot be explained by saying that the conditions vary in different cases. He concludes from the observation of twenty cases that in placenta prievia similar conditions prevail as in premature labor, that is to say, that in that condition the defective development of the supravaginal portion is a typical phenomenon; that furthermore the internal os occasionally remains closed until the actual commencement of labor, and that in that case no lower segment is formed.

Dr. Bayer distinguishes:

A. Simple low insertion of the placenta, where the margin of the placenta reaches only into the neighborhood of the internal os. Here hemorrhage occurs only during labor, when a portion of the placenta remains in the area of relaxation, $i$. $e$., when the lower segment is not formed normally. Accordingly he found low insertion of the placenta with hemorrhage during delivery only in premature labors in the eighth or ninth month.

13. 'True placenta previa. 'To constitute this condition, it is necessary that some portion of the placenta at some time covers the internal os. There is only a difference in degree between placenta previa centralis and lateralis; the former may pass into the latter in the course of pregnancy or not until labor, when the supravaginal portion develops accordingly; inversely, the symptoms of placenta pravia centralis may be present, although only a small lobe projects over the internal os, when the latter did not dilate materially before the examination. As the internal os opens, the placenta inserted upon it must become detached or it must tear; in either case hamorrhage occurs. If this takes place during pregnancy, provided the latter continues after the first hemorrhage, we must expect alterations in the placenta. In the cases observed by him, such alterations were constant when hamorrhage had occurred previous to labor; they were always absent when the first hamorrhage took place at the outset of true contractions. He distinguishes:

I. Hamorrhages during pregnancy; they characterize cases in which the os opens during pregnancy.

$a$. The placenta remains firmly on its seat, while the portion on the os internum tears; the laceration may extend to the membrana chorii; in this case labor will probably set in soon or the child die from loss of blood. If the internal os opens very gradually, more superficial lesions of the placenta may arise; according to the view of the author, a placenta marginata occasionally forms by the tearing apart of 